\title{
Komar integral and Smarr formula for axion-dilaton black holes versus S duality
}

\author{
Dimitrios Mitsios, ${ }^{a}$ Tomás Ortín $^{a}$ and David Pereñíguez ${ }^{a, b}$ \\ ${ }^{a}$ Instituto de Física Teórica UAM/CSIC, \\ C/ Nicolás Cabrera, 13-15, C.U. Cantoblanco, E-28049 Madrid, Spain \\ ${ }^{b}$ CENTRA, Departamento de Física, Instituto Superior Técnico, Universidade de Lisboa, \\ Avenida Rovisco Pais 1, 1049 Lisboa, Portugal \\ E-mail: di.mitsios@gmail.com, tomas.ortin@csic.es, \\ david.perenniguez@uam.es
}

ABSTRACT: We construct the Komar integral for axion-dilaton gravity using Wald's formalism and momentum maps and we use it to derive a Smarr relation for stationary axiondilaton black holes. While the Wald-Noether 2-form charge is not invariant under $\operatorname{SL}(2, \mathbb{R})$ electric-magnetic duality transformations because Wald's formalism does not account for magnetic charges and potentials, the Komar integral constructed with it turns out to be invariant and, in more general theories, it will be fully symplectic invariant. We check the Smarr formula obtained with the most general family of static axion-dilaton black holes.

Keywords: Black Holes, Classical Theories of Gravity, Supersymmetry and Duality

ARXiv EPrint: 2106.07495 


\section{Contents}

1 Introduction 1

2 Axion-dilaton gravity 3

3 Static dilaton-axion black hole solutions 4

4 Komar integral $\quad 6$

5 Checking the Smarr formula for static axion-dilaton black holes $\quad 9$

6 Charges, potentials and S duality 10

$\begin{array}{lll}7 & \text { Discussion } & 12\end{array}$

\section{Introduction}

In refs. [1-3] Wald and his collaborators Lee and Iyer constructed a powerful formalism that could be used to prove the first law of black-hole mechanics [4] and, through this proof, to find the entropy formula for black-hole solutions of any diffeomorphism-invariant theory. This formalism has been very successful in absence of matter fields but it was not clear how to use it on their presence. It is known that, in many cases, these fields give rise to new terms in the first law, associated to the possible variations of the conserved charges associated to them. It was unclear how these terms could arise in this formalism since it is based in diffeomorphism invariance alone and, apparently, the gauge symmetries that ensure the conservation of the charges that occur in the additional terms of the first law play no rôle whatsoever.

As we have discussed in refs. [5-7], diffeomorphisms and gauge transformations are, actually, closely related, because gauge fields are not just tensors. This was one of the main assumptions in the derivation of the well-known Iyer-Wald prescription for the entropy ref. [3]. The transformation of a gauge field under an isometry which leaves invariant all the fields of a black-hole solution always induces a gauge transformation, which, when correctly taken into account [8] (via covariant Lie derivatives, for instance), gives rise to the missing terms in the first law. If one uses a tetrad formulation, although the Vielbein is not a matter field, one must properly take into account that it transforms under local Lorentz transformations as well [9] using the Lie-Lorentz covariant derivative (see refs. [10-12] and references therein).

Still, terms associated to the variations of charges which are not associated to gauge symmetries, such as magnetic charges, will not appear derivations of the first law based on Wald's formalism, while they are known to appear in other derivations of the first law [13]. 
Terms associated to the variations of the asymptotic values of the scalars (moduli) such as those found in ref. [14] (see, also, ref. [15]), will not appear, either. This fact does not invalidate the first law, but it is a limitation to its applicability since one cannot study the effects of the variations of the missing charges.

Smarr formulae [16] provide another approach to this problem. They are closely related to the first law: the scaling arguments of refs. [17, 18] show how the thermodynamical variables (typically, charges) and their conjugate thermodynamical potentials must occur in the Smarr formula. This argument explains why there are no terms associated to the moduli in the first law if one accepts that the black-hole mass does not depend on them when it is expressed in terms of the entropy and the conserved charges. ${ }^{1}$

If the black holes under consideration have magnetic charges, then their Smarr formula must contain a term proportional to them and their associated potentials.

As explained in refs. [17, 18], Smarr formulae can be derived from Komar integrals [21]. In ref. [22] it was shown how to construct Komar integrals in general theories using Wald's formalism. The integrand contains a surface term which is the Noether-Wald charge and a volume term proportional to the on-shell Lagrangian density. As shown in ref. [23], the volume term can always be expressed as a surface term. Since the variation of the integral the Noether-Wald charge gives the first law without variations of magnetic charges and since, as we have argued, the Smarr formula must contain terms with magnetic charges and potentials, it is not clear how and if those terms are going to appear. Moreover, electric and magnetic terms must occur in an electric-magnetic symmetric form in the Smarr formula if the equations of motion of the theory have that property.

In this paper we want to study if and how this electric-magnetic duality invariance of the Smarr formula arises from a formalism (Wald's) which is not electric-magnetic symmetric because only the gauge transformations which imply the conservation of the electric charges are taken into account. To this order, in section 2, we are going to study the static black-hole solutions of a 4-dimensional theory whose equations of motion are invariant under the archetype of electric-magnetic (or S-) duality group: "axion-dilaton gravity," which is the bosonic sector of pure, ungauged, $\mathcal{N}=4, d=4$ supergravity [24]. The family of solutions that we are going to study, found in ref. [25] is invariant, as a family, under the $\mathrm{SL}(2, \mathbb{Z})$ duality group and the results obtained should be automatically invariant under that group. These solutions will be introduced in section 3 . In section 4 we will construct the Komar integral as a surface integral in a manifestly gauge and diffeomorphism-covariant form using the momentum maps introduced in refs. [5-7]. In section 5 we will use the Komar integral to explicitly test the Smarr formula for the static axion-dilaton black holes under consideration. A general form of the Smarr formula will, then, be given in section 6 , where we will discuss its electric-magnetic $\operatorname{SL}(2, \mathbb{R})$ invariance. Finally, section 7 contains our conclusions and some directions for future work.

\footnotetext{
${ }^{1}$ This fact follows from the independence of the entropy on the moduli, which, to the best of our knowledge, has been proven for static, extremal, asymptotically-flat black holes only [19, 20].
} 


\section{Axion-dilaton gravity}

The 4-dimensional model known as "axion-dilaton gravity" is nothing but the bosonic sector of pure, ungauged, $\mathcal{N}=4, d=4$ supergravity [24] and describes two scalars: the axion $a$ and the dilaton $\phi$ combined into the complex axidilaton field $\lambda \equiv a+i e^{-2 \phi}$ (often denoted by $\tau$ ) that parametrizes the coset space $\mathrm{SL}(2, \mathbb{R}) / \mathrm{SO}(2)$, and six 1-form fields $A^{m}=A^{m}{ }_{\mu} d x^{\mu}$ with 2-form field strengths

$$
F^{m}=d A^{m},
$$

coupled to gravity, which we will describe through the Vierbein $e^{a}=e^{a}{ }_{\mu} d x^{\mu}$. The number of 1-forms does not play a relevant rôle if it is larger than one, and can be left undetermined although it has to be set to six if one wants to embed the solutions of the theory into the Heterotic Superstring (HST) effective action compactified on a $T^{6}$. The model with just two 1 -forms can also be viewed as a model of $\mathcal{N}=2, d=4$ supergravity coupled to a single vector multiplet, and one can use the powerful solution-generating techniques developed in that class of models to construct extremal $[26,27]$ and non-extremal $[27,28]$ blackhole solutions.

The action of the theory in the conventions of ref. $[29]^{2}$ in differential-form language is (summation over repeated $m$ indices is understood)

$$
\begin{aligned}
S=\frac{1}{16 \pi G_{N}^{(4)}} \int\left[-\star\left(e^{a} \wedge e^{b}\right) \wedge R_{a b}+2 d \phi \wedge \star d \phi+\frac{1}{2} e^{4 \phi} d a \wedge \star d a\right. \\
\\
\left.+2 e^{-2 \phi} F^{m} \wedge \star F^{m}+2 a F^{m} \wedge F^{m}\right] \\
\equiv \int \mathbf{L} .
\end{aligned}
$$

We will set $G_{N}^{(4)}=1$ and we will ignore the normalization factor $(16 \pi)^{-1}$ for the time being.

The equations of motion are defined by

$$
\delta S=\int\left\{\mathbf{E}_{a} \wedge \delta e^{a}+\mathbf{E}_{\phi} \delta \phi+\mathbf{E}_{(a)} \delta a+\mathbf{E}_{m} \wedge A^{m}+d \boldsymbol{\Theta}(\varphi, \delta \varphi)\right\},
$$

and given by

$$
\begin{aligned}
\mathbf{E}_{a}= & \imath_{a} \star\left(e^{b} \wedge e^{c}\right) \wedge R_{b c}+2\left(\imath_{a} d \phi \star d \phi+d \phi \wedge \imath_{a} \star d \phi\right) \\
& +\frac{1}{2} e^{4 \phi}\left(\imath_{a} d a \star d a+d a \wedge \imath_{a} \star d a\right)+2 e^{-2 \phi}\left(\imath_{a} F^{m} \wedge \star F^{m}-F^{m} \wedge \imath_{a} \star F^{m}\right), \\
\mathbf{E}_{\phi}= & -4 d \star d \phi+2 e^{4 \phi} d a \wedge \star d a-4 e^{-2 \phi} F^{m} \wedge \star F^{m}, \\
\mathbf{E}_{(a)}= & -d\left(e^{4 \phi} \star d a\right)+2 F^{m} \wedge F^{m}, \\
\mathbf{E}_{m}= & -4 d F_{m}
\end{aligned}
$$

${ }^{2}$ The only difference with the conventions of refs. [25, 30-33] is that no imaginary units are introduced with the Hodge dualization. These conventions are the same used in refs. [5-7]. 
where we have defined the dual 2-form field strength

$$
F_{m} \equiv \frac{1}{4} \frac{\delta S}{\delta F^{m}}=e^{-2 \phi} \star F^{m}+a F^{m}
$$

Furthermore,

$$
\boldsymbol{\Theta}(\varphi, \delta \varphi)=-\star\left(e^{a} \wedge e^{b}\right) \wedge \delta \omega_{a b}+4 \star d \phi \delta \phi+e^{4 \phi} \star d a \delta a+4 F_{m} \wedge \delta A^{m}
$$

Since the Maxwell equations tell us that the $F_{m}$ s are closed on-shell, we can introduce a dual 1-form field $A_{m}$ defined by

$$
F_{m}=d A_{m}
$$

\section{Static dilaton-axion black hole solutions}

The most general family of non-extremal, static, black holes with non-trivial dilaton, axion and electromagnetic fields was obtained in ref. [25]. ${ }^{3}$ In the notation of ref. [29], these solutions take the form ${ }^{4}$

$$
\begin{aligned}
d s^{2} & =e^{2 U} d t^{2}-e^{-2 U} d r^{2}-R^{2} d \Omega_{(2)}^{2}, \\
\lambda & =\frac{\lambda_{\infty} r+\lambda_{\infty}^{*} \Upsilon}{r+\Upsilon}, \\
A^{m}{ }_{t} & =e^{\phi_{\infty}} R^{-2}\left[\Gamma^{m}(r+\Upsilon)+\text { c.c. }\right], \\
A_{m t} & =e^{\phi_{\infty}} R^{-2}\left[\Gamma^{m}\left(\lambda_{\infty} r+\lambda_{\infty}^{*} \Upsilon\right)+\text { c.c. }\right],
\end{aligned}
$$

where the functions that occur in the metric are

$$
\begin{aligned}
e^{2 U} & =R^{-2}\left(r-r_{+}\right)\left(r-r_{-}\right), & & r_{ \pm}=M \pm r_{0} \\
R^{2} & =r^{2}-|\Upsilon|^{2}, & r_{0}^{2} & =M^{2}+|\Upsilon|^{2}-4 \Gamma^{m} \Gamma^{m *}
\end{aligned}
$$

In these functions, $M$ is the ADM mass, the constants $\Gamma^{m}$ are related to the complex electromagnetic charges, $\lambda_{\infty}=a_{\infty}+i e^{-2 \phi_{\infty}}$ is the asymptotic value of the axidilaton and $\Upsilon=\Sigma+i \Delta$ is the axidilaton charge. All these parameters are defined by the asymptotic

\footnotetext{
${ }^{3}$ These solutions were obtained by an $\mathrm{SL}(2, \mathbb{R})$ rotation of those found in ref. [31]. The case with a single 1-form had been dealt with in ref. [34], but it is qualitatively different since these solutions can have electric and magnetic charges and vanishing axion. In their turn, the solutions of ref. [31] are a generalization of those in ref. [30], which were originally discovered by Gibbons and Maeda in refs. [35, 36]. The single-vector case was rediscovered by Garfinkle, Horowitz and Strominger in ref. [37] and it is the solution on which the $\mathrm{SL}(2, \mathbb{R})$ rotation was performed in ref. [34]. Stationary generalizations (inclusion of NUT charge) were constructed in [32] and, for the extremal case, using supersymmetry and spinorial techniques, in ref. [38] (see also ref. [33].) Finally, the most general, non-extremal, stationary black-hole solution of the model was constructed in ref. [29].

${ }^{4}$ This presentation of the solutions uses only the time components of the original and dual vector fields. As we are going to see, this information is enough to fully reconstruct all the components of these vectors.
} 
expansions

$$
\begin{aligned}
g_{t t} & \sim 1-\frac{2 M}{r}, \\
\lambda & \sim \lambda_{\infty}-i e^{-2 \phi_{\infty}} \frac{2 \Upsilon}{r}, \\
\frac{1}{2}\left[F^{m}{ }_{t r}+i \star F^{m}{ }_{t r}\right] & \sim \frac{e^{+\phi_{\infty} \Gamma^{m}}}{r^{2}}=\frac{e^{+\phi_{\infty}}\left(Q^{m}+i P^{m}\right) / 2}{r^{2}} .
\end{aligned}
$$

The axidilaton charge is not an independent parameter. In accordance with the no-hair theorem, it is a function of the ADM mass and the electric and magnetic charges

$$
\Upsilon=-\frac{2}{M} \Gamma^{m *} \Gamma^{m *} .
$$

The singularity is hidden under a horizon located at $r=r_{+}$if $r_{0}^{2}>0$, and it is hidden or coincides with it (but still is invisible for external observers) if $r_{0}=0$.

The solution has been expressed, by convenience, using only the electric components of the 1-forms and the dual 1-forms. The magnetic components can be obtained as follows. From the definition of the dual 2-form field strengths eq. (2.5), we get

$$
F_{m r t}=\frac{e^{-2 \phi}}{R^{2} \sin \theta} F_{\theta \varphi}^{m}+a F_{r t}^{m},
$$

so

$$
F_{\theta \varphi}^{m}=e^{2 \phi} R^{2} \sin \theta\left(F_{m r t}-a F^{m}{ }_{r t}\right)=2 e^{\phi_{\infty}} \Im \mathrm{m}\left(\Gamma^{m}\right) \sin \theta .
$$

The gauge field $A^{m}$, then, has to be defined in two patches. On the $z \geq-\epsilon$ patch it is given by the 1 -form

$$
A^{m+}=e^{\phi_{\infty}} R^{-2}\left[\Gamma^{m}(r+\Upsilon)+\text { c.c. }\right] d t+2 e^{\phi_{\infty}} \Im \mathrm{m}\left(\Gamma^{m}\right)(1-\cos \theta) d \varphi,
$$

which is regular in that region ${ }^{5}$ and in the $z \leq+\epsilon$ patch, it is given by the 1 -form

$$
A^{m-}=e^{\phi_{\infty}} R^{-2}\left[\Gamma^{m}(r+\Upsilon)+\text { c.c. }\right] d t-2 e^{\phi_{\infty}} \Im \mathrm{m}\left(\Gamma^{m}\right)(1+\cos \theta) d \varphi,
$$

which is also regular in that patch. $A^{m+}$ and $A^{m-}$ differ by the gauge transformation

$$
A^{m+}-A^{m-}=d\left[4 e^{\phi_{\infty}} \Im \mathrm{m}\left(\Gamma^{m}\right) \varphi\right] .
$$

We can also compute the complete dual vector fields. From the definition eq. (2.5) we find that

$$
F_{m \theta \varphi}=e^{-2 \phi} R^{2} \sin \theta F_{t r}^{m}+a F^{m}{ }_{\theta \varphi}=2 e^{\phi_{\infty}}\left\{e^{-2 \phi_{\infty}} \Re \mathrm{e}\left(\Gamma^{m}\right)+a_{\infty} \Im \mathrm{m}\left(\Gamma^{m}\right)\right\} \sin \theta,
$$

and

$$
\begin{aligned}
& A_{m}{ }^{+}=e^{\phi_{\infty}} R^{-2}\left[\Gamma^{m}\left(\lambda_{\infty} r+\lambda_{\infty}^{*} \Upsilon\right)+\text { c.c. }\right] d t
\end{aligned}
$$

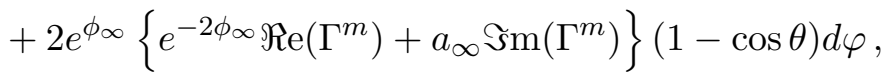

$$
\begin{aligned}
& A_{m}{ }^{-}=e^{\phi_{\infty}} R^{-2}\left[\Gamma^{m}\left(\lambda_{\infty} r+\lambda_{\infty}^{*} \Upsilon\right)+\text { c.c. }\right] d t
\end{aligned}
$$

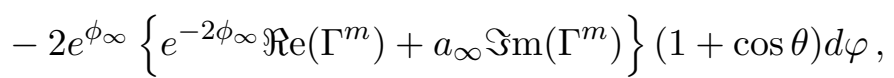

\footnotetext{
${ }^{5}$ The Dirac string singularity of this 1-form lies in the negative $z$ axis.
} 
in the same two patches, and

$$
A_{m}{ }^{+}-A_{m}{ }^{-}=d\left\{4 e^{\phi_{\infty}}\left[e^{-2 \phi_{\infty}} \Re \mathrm{e}\left(\Gamma^{m}\right)+a_{\infty} \Im \mathrm{m}\left(\Gamma^{m}\right)\right] \varphi\right\} .
$$

The Hawking temperature and Bekenstein-Hawking entropy of these black holes are given by

$$
\begin{aligned}
T & =\frac{1}{4 \pi} \partial_{r} g_{t t}\left(r_{+}\right)=\frac{r_{0}}{2 \pi R^{2}\left(r_{+}\right)}, \\
S & =\pi R^{2}\left(r_{+}\right) .
\end{aligned}
$$

Observe that, as usual in 4-dimensional, static black holes

$$
2 S T=r_{0} .
$$

Then, it is not difficult to find a Smarr-type relation adding the ADM mass to the above relation:

$$
\begin{aligned}
M & =2 S T+M-r_{0}=2 S T+r_{-}=2 S T+\frac{r_{-} r_{+}}{r_{+}}=2 S T+\frac{M^{2}-r_{0}^{2}}{r_{+}} \\
& =2 S T+\frac{4 \Gamma^{m} \Gamma^{m *}-|\Upsilon|^{2}}{r_{+}}=2 S T+\frac{Q^{m}}{r_{+}} Q^{m}+\frac{P^{m}}{r_{+}} P^{m}-\frac{\Sigma}{r_{+}} \Sigma-\frac{\Delta}{r_{+}} \Delta .
\end{aligned}
$$

This relation is correct (by construction) and, looking at it, it is tempting to conclude that the $1 / r_{+}$terms (that include those associated to the scalar charges) can immediately be identified with potentials on the horizon. However, as we are going to see, $r_{-}$can be rewritten in other ways in which only potentials associated to the electric and magnetic charges occur. Note that the usual scaling argument does not allow for terms including scalar charges or potentials because, by the no-hair theorem, these cannot be independent. Indeed, the Komar charge leaves only room for electric and magnetic potentials and charges, and, as we are going to see, the integral gives the above relation, although in a highly nontrivial way.

\section{Komar integral}

As explained, for instance, in refs. [17, 18] Smarr formulae [16] can be systematically obtained from Komar integrals [21]. These can be constructed using Wald's formalism following ref. [22], rewriting the volume integral terms as surface terms as explained in ref. [23]. In that reference, though, the integrand of the surface integral was determined after explicit evaluation of the Lagrangian density on a particular family of solutions. Here we are going to show how that integrand can be found in general. ${ }^{6}$

Let us review the construction of the Komar charge and integral in refs. [22, 23]. It is not difficult to see that, on-shell ${ }^{7}$ and for a Killing vector $k$ that generates a symmetry of the whole field configuration

$$
\mathbf{J}[k] \doteq \iota_{k} \mathbf{L} .
$$

\footnotetext{
${ }^{6}$ It is assumed, though, that we are restricting ourselves to solutions admitting a timelike Killing vector with a Killing horizon.

${ }^{7}$ We are going to use the symbol $\doteq$ for identities that only hold on-shell.
} 
On the other hand, for any vector field $\xi$, we have the off-shell (local) identity

$$
\mathbf{J}[\xi]=d \mathbf{Q}[\xi] .
$$

Combining these two relations, we find that, on-shell and for a Killing vector $k$ that generates a symmetry of the whole field configuration

$$
d \mathbf{Q}[k]-\imath_{k} \mathbf{L} \doteq 0 .
$$

However, if $k$ generates a symmetry of the whole field configuration,

$$
0 \doteq £_{k} \mathbf{L}=d \imath_{k} \mathbf{L},
$$

which implies the local existence of a $(d-2)$-form $\omega_{k}$ such that

$$
d \omega_{k} \doteq \imath_{k} \mathbf{L} .
$$

It follows that, under the aforementioned conditions,

$$
d\left\{\mathbf{Q}[k]-\omega_{k}\right\} \doteq 0 .
$$

and we can define the Komar integral over the codimension-2 surface $\Sigma^{d-2}$ ref. [23]

$$
\mathcal{K}\left(\Sigma^{d-2}\right)=(-1)^{d-1} \int_{\Sigma^{d-2}}\left\{\mathbf{Q}[k]-\omega_{k}\right\}
$$

Smarr formulae for black-hole spacetimes are obtained by integrating the identity eq. (4.6) on hypersurfaces $\Sigma$ with boundaries at the horizon and spatial infinity $\partial \Sigma_{h}$ (usually, the bifurcation surface) and $\partial \Sigma_{\infty}$, respectively upon use of Stokes theorem:

$$
\mathcal{K}\left(\partial \Sigma_{\infty}\right)=\mathcal{K}\left(\partial \Sigma_{h}\right)
$$

Using the techniques developed in refs. [5-7] and some of the results found in them, we can readily find the Noether-Wald charge for axion-dilaton gravity:

$$
\mathbf{Q}[\xi]=\star\left(e^{a} \wedge e^{b}\right) P_{\xi a b}-4 P^{m} F_{m} .
$$

Here,

$$
P_{\xi a b}=\nabla_{[a} \xi_{b]} \cdot
$$

Also, the functions $P^{m} \xi$ can be understood as the parameters of compensating gauge transformations of the 1 -forms with the property that, when $\xi=k$, they satisfy the relations

$$
d P_{k}^{m}=-\imath_{k} F^{m},
$$

that define the momentum maps associated to the Killing vector $k$ and the gauge fields $A^{m}$. Although this is a gauge-invariant definition, these objects are defined up to an additive constant. Since they can be interpreted as electrostatic potentials, the constant can be determined by a sensible boundary condition, such as the vanishing of the potentials at spatial infinity. 
In order to compute $\omega_{k}$, we have to determine the on-shell value of the Lagrangian density $\mathbf{L}$ first, for a generic solution. In this case, it is enough to use the trace of the Einstein equations eqs. (2.4a). In differential-form language, to take the trace we must compute $e^{a} \wedge \mathbf{E}_{a}$, taking into account that, for a $p$-form $\omega^{(p)}$,

$$
e^{a} \wedge \imath_{a} \omega^{(p)}=p \omega^{(p)}
$$

We get

$$
\begin{aligned}
e^{a} \wedge \mathbf{E}_{a} & =-2\left\{-\star\left(e^{c} \wedge e^{d}\right) \wedge R_{c d}+2 d \phi \wedge \star d \phi+\frac{1}{2} e^{4 \phi} d a \wedge \star d a\right\} \\
& =-2\left\{\mathbf{L}-2 e^{-2 \phi} F^{m} \wedge \star F^{m}-2 a F^{m} \wedge F^{m}\right\}
\end{aligned}
$$

SO

$$
\mathbf{L} \doteq 2 e^{-2 \phi} F^{m} \wedge \star F^{m}+2 a F^{m} \wedge F^{m}=2 F^{m} \wedge F_{m},
$$

and

$$
\imath_{k} \mathbf{L} \doteq 2 \imath_{k} F^{m} \wedge F_{m}+2 F^{m} \wedge \imath_{k} F_{m} .
$$

In order to find $\omega_{k}$ for general configurations, we are going to use the definition of the (electric) momentum maps eq. (4.11) but we need to define their magnetic duals. Since, by assumption, the dual field strengths are left invariant by the isometry generated by $k$,

$$
0=£_{k} F_{m}=d \imath_{k} F_{m}+\imath_{k} d F_{m} \doteq d \imath_{k} F_{m}
$$

where we have used the Maxwell equations. Then, locally, there are functions $P_{m k}$ (magnetic momentum maps) such that

$$
d P_{m k} \doteq-\imath_{k} F_{m}
$$

Thus, upon use of the Maxwell equations and Bianchi identities,

$$
\imath_{k} \mathbf{L} \doteq-2 d P^{m}{ }_{k} \wedge F_{m}-2 F^{m} \wedge d P_{m k} \doteq d\left\{-2 P^{m}{ }_{k} F_{m}-2 F^{m} P_{m k}\right\}=d \omega_{k},
$$

and the Komar charge is given by

$$
\mathbf{Q}[k]-\omega_{k}=\star\left(e^{a} \wedge e^{b}\right) P_{k a b}-2\left(P_{k}^{m} F_{m}-P_{m k} F^{m}\right) .
$$

Observe that the electromagnetic terms occur in a symplectic-invariant combination now. This hints at the electric-magnetic $(\operatorname{SL}(2, \mathbb{R}))$ invariance of the Komar charge, a fact that we will study in section 6 . Before studying this invariance, we are going to check the validity of this formula in the family of static black holes introduced in section 3 by direct computation of the Komar integral. 


\section{Checking the Smarr formula for static axion-dilaton black holes}

Now we want to compute the Komar integrals over the bifurcation sphere on the horizon and over a sphere at spatial infinity for the static axion-dilaton black holes introduced in section 3. Thus, we are interested in the $\theta \varphi$ components of the integrand only. We compute them term by term and we recover the normalization factor $(16 \pi)^{-1}$. First,

$$
\star\left(e^{a} \wedge e^{b}\right) P_{k a b}=\frac{1}{2 \sqrt{|g|}} \varepsilon_{\mu \nu \rho \sigma} \nabla^{\mu} k^{\nu} d x^{\rho} \wedge d x^{\sigma},
$$

and, for these solutions

$$
\begin{gathered}
\nabla^{\mu} k^{\nu}=\delta^{t[\mu} \delta^{\nu] r} \partial_{r} e^{2 U}, \\
\star\left(e^{a} \wedge e^{b}\right) P_{k a b}=-r^{2} \partial_{r} e^{2 U} \sin \theta d \theta \wedge d \varphi .
\end{gathered}
$$

The electric and magnetic momentum maps can be taken to be

$$
P_{k}^{m}=A_{t}^{m}, \quad P_{m k}=A_{m t},
$$

and, the second term in the Komar charge eq. (4.19) is (only $\theta \varphi$ components)

$$
\begin{aligned}
-2\left(P_{k}^{m} F_{m \theta \varphi}-P_{m k} F^{m}{ }_{\theta \varphi}\right) & =-2\left\{A^{m}{ }_{t}\left[e^{-2 \phi}\left(\star F^{m}\right)_{\theta \varphi}+a F^{m}{ }_{\theta \varphi}\right]-A_{m t} F^{m}{ }_{\theta \varphi}\right\} \\
& =\left\{2 R^{2} e^{-2 \phi} A^{m}{ }_{t} \partial_{r} A^{m}{ }_{t}+4 e^{\phi_{\infty}}\left(A_{m t}-a A^{m}{ }_{t}\right) \Im \mathrm{s}\left(\Gamma^{m}\right)\right\} \sin \theta .
\end{aligned}
$$

Integrating over a 2 -sphere of constant radius $r$, we get

$$
\mathcal{K}\left(S^{2}{ }_{r}\right)=\frac{1}{4} r^{2} \partial_{r} e^{2 U}-\frac{1}{2} R^{2} e^{-2 \phi} A^{m}{ }_{t} \partial_{r} A^{m}{ }_{t}-e^{\phi_{\infty}}\left(A_{m t}-a A^{m}{ }_{t}\right) \Im \mathrm{m}\left(\Gamma^{m}\right) .
$$

At infinity, only the first term contributes, giving

$$
\mathcal{K}\left(S^{2} \infty\right)=M / 2
$$

Over the bifurcation sphere, ${ }^{8}$ the first term gives $S T=r_{0} / 2$, but we have to evaluate carefully the second and third terms. We introduce some notation:

$$
A \equiv \lambda_{\infty} r+\lambda_{\infty}^{*} \Upsilon, \quad B \equiv r+\Upsilon, \quad \Rightarrow \quad \lambda=A / B
$$

The second term in eq. (5.5) is

$$
\begin{aligned}
-\frac{1}{2} R^{2} e^{-2 \phi} A^{m}{ }_{t} \partial_{r} A^{m}{ }_{t}= & \frac{1}{2 R^{2}|r+\Upsilon|^{2}}\left[\Gamma^{m} B+\text { c.c }\right]\left[-2|r+\Upsilon|^{2} \Re \mathrm{e}\left(\Gamma^{m}\right)+4 \Im \mathrm{m}\left(\Gamma^{m}\right) \Im \mathrm{m}(\Upsilon) r\right] \\
= & \frac{1}{2 R^{2}}\left[\Gamma^{m}\left(\Gamma^{m}+\Gamma^{m *}\right) B+\text { c.c }\right] \\
& +\frac{r}{R^{2}|r+\Upsilon|^{2}}\left[i \Gamma^{m}\left(\Gamma^{m}-\Gamma^{m *}\right) B+\text { c.c }\right] \Im \mathrm{m}(\Upsilon) .
\end{aligned}
$$

\footnotetext{
${ }^{8}$ Actually, it is enough to set $r=r_{+}$.
} 
Using the relation eq. (3.4) it is not hard to see that at $r=r_{+}$

$$
i \Gamma^{m}\left(\Gamma^{m}-\Gamma^{m *}\right) B\left(r_{+}\right)+\text {c.c }=-\frac{1}{2} R^{2}\left(r_{+}\right) \Im \mathrm{m}(\Upsilon) .
$$

Then,

$$
\begin{aligned}
-\left.\frac{1}{2} R^{2} e^{-2 \phi} A^{m}{ }_{t} \partial_{r} A^{m}{ }_{t}\right|_{r_{+}} & =\frac{2|\Gamma|^{2} r_{+}-M|\Upsilon|^{2}}{2 R^{2}\left(r_{+}\right)}-\frac{\left(M r_{+}-2|\Gamma|^{2}\right)}{2 R^{2}\left(r_{+}\right)} \Re \mathrm{e}(\Upsilon)-\frac{r_{+}[\Im \mathrm{m}(\Upsilon)]^{2}}{2\left|r_{+}+\Upsilon\right|^{2}} \\
& =\frac{r_{-}}{4}+\frac{\Re \mathrm{e}(\Upsilon)}{4}-\frac{r_{+}[\Im \mathrm{m}(\Upsilon)]^{2}}{2\left|r_{+}+\Upsilon\right|^{2}}
\end{aligned}
$$

The third term in eq. (5.5) is

$$
\begin{aligned}
-e^{\phi_{\infty}}\left(A_{m t}-a A^{m}{ }_{t}\right) \Im \mathrm{m}\left(\Gamma^{m}\right) & =-\frac{e^{2 \phi_{\infty}}}{R^{2}}\left[\Gamma^{m}(A-a B)+\text { c.c. }\right] \Im \mathrm{m}\left(\Gamma^{m}\right) \\
& =-\frac{e^{-2\left(\phi-\phi_{\infty}\right)}}{R^{2}}\left[i \Gamma^{m} B+\text { c.c. }\right] \Im \mathrm{m}\left(\Gamma^{m}\right) \\
& =\frac{1}{2|r+\Upsilon|^{2}}\left[\left(M \Re \mathrm{e}(\Upsilon)+2|\Gamma|^{2}\right) r+M|\Upsilon|^{2}+2|\Gamma|^{2} \Re \mathrm{e}(\Upsilon)\right] .
\end{aligned}
$$

Combining these two partial results at $r=r_{+}$and operating, we get

$$
\begin{aligned}
& \frac{r_{-}}{4}-\frac{\Re \mathrm{e}(\Upsilon)}{4}+\frac{\left(M \Re \mathrm{e}(\Upsilon)+2|\Gamma|^{2}-[\Im \mathrm{m}(\Upsilon)]^{2}\right) r_{+}+M|\Upsilon|^{2}+2|\Gamma|^{2} \Re \mathrm{e}(\Upsilon)}{2\left|r_{+}+\Upsilon\right|^{2}} \\
= & \frac{r_{-}}{4}+\frac{\Re \mathrm{e}(\Upsilon)\left(2 M r_{+}+4|\Gamma|^{2}-\left|r_{+}+\Upsilon\right|^{2}\right)+2\left(2|\Gamma|^{2}-[\Im \mathrm{m}(\Upsilon)]^{2}\right) r_{+}+2 M|\Upsilon|^{2}}{4\left|r_{+}+\Upsilon\right|^{2}} \\
= & \frac{r_{-}}{4}+\frac{\left.\Re \mathrm{e}(\Upsilon)\left(2 M r_{+}-r_{+}^{2}+4|\Gamma|^{2}-|\Upsilon|^{2}\right)+\left.2\left(2|\Gamma|^{2}-\mid \Upsilon\right)\right|^{2}\right) r_{+}+2 M|\Upsilon|^{2}}{4\left|r_{+}+\Upsilon\right|^{2}} \\
= & \frac{r_{-}}{4}+\frac{\left.2 \Re \mathrm{e}(\Upsilon) r_{+} r_{-}+r_{+}^{2} r_{-}-\mid \Upsilon\right)\left.\right|^{2} r_{-}}{4\left|r_{+}+\Upsilon\right|^{2}} \\
= & \frac{r_{-}}{2},
\end{aligned}
$$

which gives the Smarr formula proposed in section 3, eqs. (3.15).

\section{Charges, potentials and S duality}

The static axion-dilaton black holes introduced in section 3 are the most general black holes in that class according to the no-hair theorems because they have the maximum number of independent parameters (moduli $\lambda_{\infty}$ and conserved charges $M, \Gamma^{m}$ ) allowed by it. Hence, we have proven the validity of the Smarr formula in this theory for static black holes. However, we have to rewrite it in terms of the potentials and charges.

The charges which are quantized in this theory are not the components of $\Gamma^{m}$, but

$$
\begin{aligned}
p^{m} & \equiv \frac{1}{8 \pi G_{N}^{(4)}} \int F^{m}=e^{\phi_{\infty}} \Im \mathrm{m}\left(\Gamma^{m}\right) / G_{N}^{(4)}, \\
q_{m} & \equiv \frac{1}{8 \pi G_{N}^{(4)}} \int F_{m}=e^{\phi_{\infty}}\left[e^{\left.-2 \phi_{\infty} \Re \mathrm{e}\left(\Gamma^{m}\right)+a_{\infty} \Im \mathrm{m}\left(\Gamma^{m}\right)\right] / G_{N}^{(4)} .}\right.
\end{aligned}
$$


According to the discussions in refs. [5-7], the potentials can be identified, up to a normalization factor, with the momentum maps $P_{k}^{m}$ and $P_{m k}$ evaluated over the black-hole horizon:

$$
\begin{aligned}
& \left.\Phi^{m} \equiv 2 P_{k}^{m}\right|_{r_{h}}, \\
& \left.\Phi_{m} \equiv 2 P_{m k}\right|_{r_{h}},
\end{aligned}
$$

and they are guaranteed to be constant at least over the bifurcation sphere $\mathcal{B H}$, according to the restricted, generalized zeroth laws. ${ }^{9}$ We normalize them to vanish at infinity for the asymptotically-flat solutions we are interested in.

Therefore,

$$
\begin{aligned}
& \frac{1}{16 \pi G_{N}^{(4)}} \int_{S_{\infty}^{2}} 2\left(P^{m}{ }_{k} F_{m}-P_{m k} F^{m}\right)=0, \\
& \frac{1}{16 \pi G_{N}^{(4)}} \int_{\mathcal{B H}} 2\left(P^{m}{ }_{k} F_{m}-P_{m k} F^{m}\right)=\frac{1}{2}\left(\Phi^{m} q_{m}-\Phi_{m} p^{m}\right) .
\end{aligned}
$$

On the other hand, on general grounds and in the static case,

$$
\begin{aligned}
& -\frac{1}{16 \pi G_{N}^{(4)}} \int_{S_{\infty}^{2}} \star\left(e^{a} \wedge e^{b}\right) P_{k a b}=M / 2, \\
& -\frac{1}{16 \pi G_{N}^{(4)}} \int_{\mathcal{B H}} \star\left(e^{a} \wedge e^{b}\right) P_{k a b}=S T
\end{aligned}
$$

and the Smarr formula takes the general form ${ }^{10}$

$$
M=2 S T+\Phi^{m} q_{m}-\Phi_{m} p^{m} .
$$

While our definitions of charges and potentials seem to be identical to those in refs. [13, 14], we get a different sign for the last term. The scaling arguments explained in refs. [17, 18] indicate that the sign should be a plus if we define $\Phi^{m}=\partial M / \partial q_{m}$. We can always add a sign to our definition of $\Phi_{m}$ to make it coincide with that definition, but we are going to argue that a relative minus sign between the last two terms is the natural sign if we take into account that the Smarr formula should be invariant under the dualities of the theory. These always act on the vector fields of a 4-dimensional theory through a symplectic embedding [40].

In this particular case, it is convenient to define the symplectic vector of field strengths as follows:

$$
\left(\mathcal{F}^{M}\right) \equiv\left(\begin{array}{c}
F_{m} \\
F^{m}
\end{array}\right)
$$

since the action of a $\mathrm{SL}(2, \mathbb{R}) \sim \mathrm{Sp}(2, \mathbb{R})$ duality transformation

$$
S \equiv\left(S_{N}^{M}\right)=\left(\begin{array}{ll}
\alpha & \beta \\
\gamma & \delta
\end{array}\right)
$$

\footnotetext{
${ }^{9}$ This result may be extended to the complete event horizon using the arguments in ref. [41].

${ }^{10}$ A previous derivation of a Smarr formula in this theory was made in ref. [39] and our results should be compared with those in that reference.
} 
on them and on the axidilaton takes a simpler form:

$$
\mathcal{F}^{M}=S_{N}^{M} \mathcal{F}^{N}, \quad \lambda^{\prime}=\frac{\alpha \lambda+\beta}{\gamma \lambda+\delta}, \quad \alpha \delta-\beta \gamma=1 .
$$

It follows from the definitions that

$$
\left(\mathcal{P}_{k}^{M}\right) \equiv\left(\begin{array}{c}
P_{m k} \\
P_{k}^{m}
\end{array}\right), \quad\left(\Phi^{M}\right) \equiv\left(\begin{array}{c}
\Phi_{m} \\
\Phi^{m}
\end{array}\right), \quad\left(\mathcal{Q}^{M}\right) \equiv\left(\begin{array}{c}
q_{m} \\
p^{m}
\end{array}\right),
$$

transform as the $\mathrm{SL}(2, \mathbb{R})$ vector $\mathcal{F}^{M}$.

An important property of the duality group $\mathrm{SL}(2, \mathbb{R})$ is that it is isomorphic to $\operatorname{Sp}(2, \mathbb{R})$ since the condition

$$
S^{M}{ }_{P} \Omega_{M N} S^{N}{ }_{Q}=\Omega_{P Q}, \quad\left(\Omega_{M N}\right)=\left(\begin{array}{cc}
0 & 1 \\
-1 & 0
\end{array}\right),
$$

also implies $\alpha \delta-\beta \gamma=1$ for the matrix $S$. Thus, the combination of potentials and charges occurring in the Smarr formula eq. (6.5)

$$
\Phi^{m} q_{m}-\Phi_{m} p^{m}=\mathcal{Q}^{M} \Phi^{N} \Omega_{M N}
$$

is manifestly $\mathrm{SL}(2, \mathbb{R}) \sim \mathrm{Sp}(2, \mathbb{R})$-invariant. The explicit calculation of this term in section 5 is a proof of this invariance.

\section{Discussion}

In this paper we have shown how the momentum maps introduced in refs. [5-7] in the context of black-hole thermodynamics can be used to express the Komar integral obtained in the context of Wald's formalism [22] as a surface integral in a manifestly covariant way, generalizing the results of $[17,18,23]$. We have also shown how, in its turn, this integral can be used to derive a Smarr formula which is manifestly symplectic invariant. We have checked this formula explicitly in the most general family of static axidilaton black holes, constructed in ref. [25]. It is trivial to extend these results to theories with more scalars and more complicated kinetic matrices (period matrices in the language of $\mathcal{N}=2$ theories).

Symplectic invariance is a property to be expected of a general Smarr formula because this relation is just a relation between physical parameters occurring in the metric, which is symplectic invariant. It is, nevertheless, surprising, how this property of the Smarr formula and of the Komar integral from which it is derived, arises from a combination of the Noether-Wald charge and the on-shell Lagrangian density which are not separately symplectic invariant. The lack of symplectic invariance of the Noether-Wald charge leads to a non-invariant first law ref. [5] in which the terms containing the variations of the magnetic charges are not present. This was to be expected because Wald's formalism is based on gauge symmetries and there is no gauge symmetry associated to the conservation of magnetic charges (at least in the standard, off-shell, formulation of electromagnetism and its generalizations). It is, nevertheless, somewhat unsatisfactory. ${ }^{11}$ It is also somewhat

\footnotetext{
${ }^{11}$ It is also problematic because this is the only formalism that can be applied to theories of higher order in the curvature.
} 
unsatisfactory that there is no natural place in Wald's first law for terms proportional to the variations of the asymptotic values of scalars [14] since these are not associated to gauge symmetries either. Work on these problems is in progress.

\section{Acknowledgments}

This work has been supported in part by the MCIU, AEI, FEDER (UE) grant PGC2018095205-B-I00 "Gravity, Supergravity and Superstrings" (GRASS) and by the Spanish Research Agency (Agencia Estatal de Investigación) through the grant IFT Centro de Excelencia Severo Ochoa SEV-2016-0597. The work of D.P. is supported by a "Campus de Excelencia Internacional UAM/CSIC" FPI pre-doctoral grant and the GWverse COST Action CA16104, "Gravitational waves, black holes and fundamental physics". D.P. gratefully acknowledges the hospitality and financial support of the group at CENTRA. T.O. wishes to thank M.M. Fernández for her permanent support.

Open Access. This article is distributed under the terms of the Creative Commons Attribution License (CC-BY 4.0), which permits any use, distribution and reproduction in any medium, provided the original author(s) and source are credited.

\section{References}

[1] J. Lee and R.M. Wald, Local symmetries and constraints, J. Math. Phys. 31 (1990) 725 [INSPIRE].

[2] R.M. Wald, Black hole entropy is the Noether charge, Phys. Rev. D 48 (1993) R3427 [gr-qc/9307038] [INSPIRE].

[3] V. Iyer and R.M. Wald, Some properties of Noether charge and a proposal for dynamical black hole entropy, Phys. Rev. D 50 (1994) 846 [gr-qc/9403028] [INSPIRE].

[4] J.M. Bardeen, B. Carter and S.W. Hawking, The Four laws of black hole mechanics, Commun. Math. Phys. 31 (1973) 161 [INSPIRE].

[5] Z. Elgood, P. Meessen and T. Ortín, The first law of black hole mechanics in the Einstein-Maxwell theory revisited, JHEP 09 (2020) 026 [arXiv: 2006. 02792] [INSPIRE].

[6] Z. Elgood, D. Mitsios, T. Ortín and D. Pereñíguez, The first law of heterotic stringy black hole mechanics at zeroth order in $\alpha$, JHEP 07 (2021) 007 [arXiv:2012.13323] [INSPIRE].

[7] Z. Elgood, T. Ortín and D. Pereñíguez, The first law and Wald entropy formula of heterotic stringy black holes at first order in $\alpha^{\prime}$, JHEP 05 (2021) 110 [arXiv:2012.14892] [INSPIRE].

[8] K. Prabhu, The First Law of Black Hole Mechanics for Fields with Internal Gauge Freedom, Class. Quant. Grav. 34 (2017) 035011 [arXiv:1511.00388] [INSPIRE].

[9] T. Jacobson and A. Mohd, Black hole entropy and Lorentz-diffeomorphism Noether charge, Phys. Rev. D 92 (2015) 124010 [arXiv:1507.01054] [InSPIRE].

[10] T. Ortín, A Note on Lie-Lorentz derivatives, Class. Quant. Grav. 19 (2002) L143 [hep-th/0206159] [INSPIRE].

[11] L. Fatibene and M. Francaviglia, General theory of Lie derivatives for Lorentz tensors, Comm. Math. 19 (2011) 11 [arXiv:0904.0258]. 
[12] T. Ortín, Gravity and Strings, 2nd edition, Cambridge University Press (2015) [INSPIRE].

[13] P. Breitenlohner, D. Maison and G.W. Gibbons, Four-Dimensional Black Holes from Kaluza-Klein Theories, Commun. Math. Phys. 120 (1988) 295 [INSPIRE].

[14] G.W. Gibbons, R. Kallosh and B. Kol, Moduli, scalar charges, and the first law of black hole thermodynamics, Phys. Rev. Lett. 77 (1996) 4992 [hep-th/9607108] [InSPIRE].

[15] D. Astefanesei, R. Ballesteros, D. Choque and R. Rojas, Scalar charges and the first law of black hole thermodynamics, Phys. Lett. B $\mathbf{7 8 2}$ (2018) 47 [arXiv:1803.11317] [INSPIRE].

[16] L. Smarr, Mass formula for Kerr black holes, Phys. Rev. Lett. 30 (1973) 71 [Erratum ibid. 30 (1973) 521] [INSPIRE].

[17] D. Kastor, Komar Integrals in Higher (and Lower) Derivative Gravity, Class. Quant. Grav. 25 (2008) 175007 [arXiv:0804.1832] [INSPIRE].

[18] D. Kastor, S. Ray and J. Traschen, Smarr Formula and an Extended First Law for Lovelock Gravity, Class. Quant. Grav. 27 (2010) 235014 [arXiv:1005.5053] [INSPIRE].

[19] S. Ferrara, G.W. Gibbons and R. Kallosh, Black holes and critical points in moduli space, Nucl. Phys. B 500 (1997) 75 [hep-th/9702103] [INSPIRE].

[20] A. Sen, Entropy function for heterotic black holes, JHEP 03 (2006) 008 [hep-th/0508042] [INSPIRE].

[21] A. Komar, Covariant conservation laws in general relativity, Phys. Rev. 113 (1959) 934 [INSPIRE].

[22] S. Liberati and C. Pacilio, Smarr Formula for Lovelock Black Holes: a Lagrangian approach, Phys. Rev. D 93 (2016) 084044 [arXiv:1511.05446] [INSPIRE].

[23] T. Ortín, Komar integrals for theories of higher order in the curvature and black-hole chemistry, arXiv:2104.10717 [INSPIRE].

[24] E. Cremmer, J. Scherk and S. Ferrara, SU(4) Invariant Supergravity Theory, Phys. Lett. B 74 (1978) 61 [INSPIRE].

[25] R. Kallosh and T. Ortín, Charge quantization of axion - dilaton black holes, Phys. Rev. D 48 (1993) 742 [hep-th/9302109] [INSPIRE].

[26] P. Meessen and T. Ortín, The Supersymmetric configurations of $N=2, D=4$ supergravity coupled to vector supermultiplets, Nucl. Phys. B $\mathbf{7 4 9}$ (2006) 291 [hep-th/0603099] [INSPIRE].

[27] P. Meessen, T. Ortín, J. Perz and C.S. Shahbazi, H-FGK formalism for black-hole solutions of $N=2, d=4$ and $d=5$ supergravity, Phys. Lett. B 709 (2012) 260 [arXiv:1112.3332] [INSPIRE].

[28] P. Galli, T. Ortín, J. Perz and C.S. Shahbazi, Non-extremal black holes of $N=2, d=4$ supergravity, JHEP 07 (2011) 041 [arXiv:1105.3311] [INSPIRE].

[29] E. Lozano-Tellechea and T. Ortín, The General, duality invariant family of nonBPS black hole solutions of $N=4, D=4$ supergravity, Nucl. Phys. B $\mathbf{5 6 9}$ (2000) 435 [hep-th/9910020] [INSPIRE].

[30] R. Kallosh, A.D. Linde, T. Ortín, A.W. Peet and A. Van Proeyen, Supersymmetry as a cosmic censor, Phys. Rev. D 46 (1992) 5278 [hep-th/9205027] [INSPIRE].

[31] T. Ortín, Electric-magnetic duality and supersymmetry in stringy black holes, Phys. Rev. D 47 (1993) 3136 [hep-th/9208078] [INSPIRE]. 
[32] R. Kallosh, D. Kastor, T. Ortín and T. Torma, Supersymmetry and stationary solutions in dilaton axion gravity, Phys. Rev. D 50 (1994) 6374 [hep-th/9406059] [INSPIRE].

[33] E. Bergshoeff, R. Kallosh and T. Ortín, Stationary axion/dilaton solutions and supersymmetry, Nucl. Phys. B 478 (1996) 156 [hep-th/9605059] [INSPIRE].

[34] A.D. Shapere, S. Trivedi and F. Wilczek, Dual dilaton dyons, Mod. Phys. Lett. A 6 (1991) 2677 [INSPIRE].

[35] G.W. Gibbons, Antigravitating Black Hole Solitons with Scalar Hair in $N=4$ Supergravity, Nucl. Phys. B 207 (1982) 337.

[36] G.W. Gibbons and K.-i. Maeda, Black Holes and Membranes in Higher Dimensional Theories with Dilaton Fields, Nucl. Phys. B 298 (1988) 741 [INSPIRE].

[37] D. Garfinkle, G.T. Horowitz and A. Strominger, Charged black holes in string theory, Phys. Rev. D 43 (1991) 3140 [Erratum ibid. 45 (1992) 3888] [INSPIRE].

[38] K.P. Tod, More on supercovariantly constant spinors, Class. Quant. Grav. 12 (1995) 1801 [INSPIRE].

[39] M. Rogatko, Stationary axisymmetric axion - dilaton black holes: Mass formulae, Class. Quant. Grav. 11 (1994) 689 [INSPIRE].

[40] M.K. Gaillard and B. Zumino, Duality Rotations for Interacting Fields, Nucl. Phys. B 193 (1981) 221 [INSPIRE].

[41] T. Jacobson, G. Kang and R.C. Myers, On black hole entropy, Phys. Rev. D 49 (1994) 6587 [gr-qc/9312023] [INSPIRE]. 